\title{
Young hematologists and medical oncologists in focus
}

\author{
Barbara Kiesewetter (1) for the "Young Hematologists \& Oncologists Group Austria" (YHOGA) - Andreas Pircher \\ for the "Young Hematologists \& Oncologists Group Austria" (YHOGA) - Alexandra Boehm for the "Young \\ Hematologists \& Oncologists Group Austria" (YHOGA)
}

Received: 4 December 2019 / Accepted: 10 December 2019

(C) Springer-Verlag GmbH Austria, part of Springer Nature 2020
In view of the constantly rising number of novel compounds and the increasing complexity of available treatment modalities, the spectrum of professional life in the field of oncology and malignant hematology has drastically changed in the last decade. While previously advanced malignant diseases were mainly approached by "classical" cytotoxic agents with a clearly estimated toxicity profile, novel treatments address often very specific targets in the cancer cell and tumor microenvironment accompanied by so far unknown toxicities, ranging from autoimmune reactions to complex cytokine release syndromes. In addition, not only novel agents but also completely newly engineered treatment strategies have been introduced starting from personalized treatment based on targeted sequencing panels to such outstanding concepts like chimeric antigen receptor (CAR) T-cell therapies; and while some of these approaches including the latter are currently bound to very specialized centers it is obvious that such specific concepts will require a high number of well-educated specialists in the near future.

Educating such specialists will be one of the most important challenges in the current era of our profession and whereas the above-mentioned developments

\section{B. Kiesewetter $(\bowtie)$}

Division of Oncology, Department of Medicine

I, Medical University of Vienna, Waehringer Guertel

18-20, 1090 Vienna, Austria

barbara.kiesewetter@meduniwien.ac.at

\section{A. Pircher}

Division of Hematology and Oncology, Department of Medicine V, Medical University of Innsbruck, Innsbruck, Austria

\section{A. Boehm}

Department of Medicine III, Hanusch Hospital, Vienna, Austria have potentially made the field of medical oncology and hematology more attractive to young colleagues due to an increasingly diversified work spectrum, professional life in oncology is still characterized by a high work load accompanied with an enormous dual burden of combining clinical work and scientific duties, often long work hours and finally emotional strain in dealing with vitally threatened patients. Furthermore, given the lack of comprehensive support groups and mostly short recovery time, recent surveys have attested a high risk for burnout among younger colleagues in medical oncology clearly demanding improved work-life balance concepts [1]. One key element in addressing this issue is to give the next generation a voice and establish young member committees, as implemented recently by the European Society for Medical Oncology (ESMO) [2] and the European Hematology Association (EHA) [3]. Such structured programs for young oncologists and hematologists can not only identify the specific needs of this target group but also connect "younger" and "older" professionals in mentoring systems in order to provide guidance in clinical and scientific questions and also in career planning and specific challenges as compatibility of family life and career. In addition, customized fellowships and leadership programs support individual professional advancement [4].

In analogy to this, the Young Hematologists \& Oncologists Group Austria (YHOGA) constitutes a defined taskforce within the Austrian Society of Hematology and Medical Oncology (OeGHO) with a distinct focus on the needs of young hematologists and medical oncologists in Austria, aiming to build a profound network and also guide career development by educational projects, research grants and mentoring programs on a national level [5]. Thus, emphasizing the broad facets of professional development in hematology and medical oncology we appreciate the support 
of memo to bring young Austrian hematologists and oncologists "in focus" and are proud to introduce in its current issue the first part of a series specifically designed for young hematologists and oncologists, presenting and analyzing complex cases together with an experienced supervisor. The first case presented deals with a rare case of Langerhans cell sarcoma and is reported by the young Austrian hemato-oncologist Verena Schlintl. We congratulate her on the interesting case and look forward to the continuation of this section and many outstanding national and international contributions of young colleagues in the near future.

Conflict of interest B. Kiesewetter, A. Pircher and A. Boehm declare that they have no competing interests.

\section{References}

1. Banerjee S, Califano R, Corral J, et al. Professional burnout in European young oncologists: results of the European society for medical oncology (ESMO) young oncologists committee burnout survey. Ann Oncol. 2017;28:1590-6.
2. European Society for Medical Oncology. Homepage. 2019. https:// www.esmo.org. Accessed 30 Nov 2019.

3. European Hematology Association. Homepage. 2019. https:// ehaweb.org. Accessed 30 Nov 2019.

4. Morgan G, Lambertini M, Kourie HR, et al. Career opportunities and benefits for young oncologists in the European society for medical oncology (ESMO). ESMO Open. 2016;1:e107.

5. Österreichische Gesellschaft für Hämatologie \& Medizinische Onkologie (OeGHO). Aufgaben \& Ziele. 2019. https://www.oegho.at/yhoga/. Accessed 30 Nov 2019.

Publisher's Note Springer Nature remains neutral with regard to jurisdictional claims in published maps and institutional affiliations.

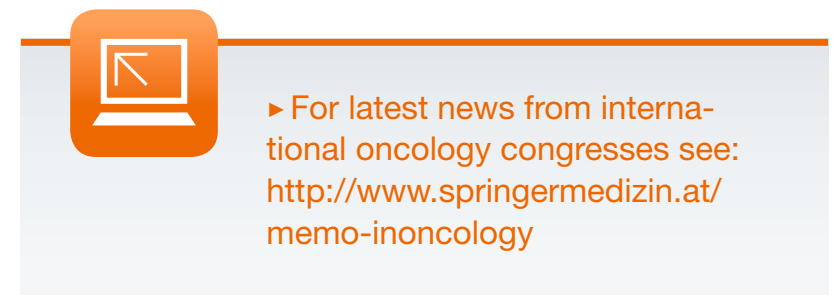

